\title{
À margem do rio e da sociedade: uma validação do instrumento de investigação das condições de saúde, socioeconômicas e ambientais em comunidades quilombolas
}

\author{
On the banks of the river and society: a validation of the instrument for investigating health, \\ socioeconomic and environmental conditions in quilombola communities
}

As oríllas del río y la sociedade: una validación del instrumento para investigar las condiciones de salud, socioeconómicas y ambientales en las comunidades quilombolas

\section{Resumo}

As comunidades quilombolas são consideradas grupos vulneráveis que necessitam de ações voltadas à realidade com o intuito de melhorar a qualidade de vida desta população. O objetivo desse estudo foi elaborar e validar um instrumento de investigação que permita analisar a associação das principais doenças relacionadas a água e a COVID19 em comunidades quilombolas, bem como conhecer o perfil socioeconômico e ambiental deste grupo populacional. Foram conduzidos testes rigorosos de avaliação metodológica de validação de conteúdo. Inicialmente, a primeira versão do instrumento contendo 79 questões foi elaborada a partir da revisão da literatura e submetida a avaliação por cinco especialistas que possuem experiências em atividades desenvolvidas com quilombolas. Após as correções e validação dos especialistas, a segunda versão com 68 questões foi aplicada como pré-teste com 50 indivíduos. A validade da versão final do instrumento foi considerada satisfatória e configura uma ferramenta útil na investigação das condições de saúde, socioeconômicas e ambientais em comunidades quilombolas.

Palavras-chave: Questionário; Populações vulneráveis; Grupo com ancestrais do Continente Africano.

\begin{abstract}
Quilombola communities are considered vulnerable groups and need actions focused on reality in order to improve the quality of life of this population. The objective of this study is to develop and validate an investigation instrument that allows analyzing the association of the main diseases related to water and COVID-19 in quilombola communities, as well as knowing the socioeconomic and environmental profile of this population group. Stringent content validation methodological evaluation tests were conducted. Initially, the first version of the instrument containing 79 questions was created based on a review of the literature and submitted to evaluation by five experts who have experience in activities developed with quilombolas. After corrections and validation by experts, the second version with 68 questions was applied as a pre-test with 50 individuals. The validity of the final version of the instrument was considered satisfactory and constitutes a useful tool in the investigation of health, socioeconomic and environmental conditions in quilombola communities.
\end{abstract}

Keywords: Questionnaire; Vulnerable Populations; African Continental ancestry group.

\section{Resumen}

Las comunidades quilombolas son consideradas grupos vulnerables que necesitan acciones enfocadas en la realidad para mejorar la calidad de vida de esta población. El objetivo de este estudio fue desarrollar y validar un instrumento de investigación que permita analizar la asociación de las principales enfermedades relacionadas con el agua y COVID-19 en comunidades quilombolas, así como conocer el perfil socioeconómico y ambiental de este grupo poblacional. Se realizaron rigurosas pruebas de evaluación metodológica de validación de contenido. Inicialmente, la primera versión del instrumento que contiene 79 preguntas fue desarrollada a partir de una revisión de la literatura y sometida a evaluación por cinco expertos que tienen experiencia en actividades desarrolladas con quilombolas. Luego 
de correcciones y validaciones por expertos, se aplicó la segunda versión con 68 preguntas como pre-test con 50 personas. La validez de la versión final del instrumento se consideró satisfactoria y constituye una herramienta útil en la investigación de las condiciones de salud, socioeconómicas y ambientales en comunidades quilombolas.

Palabras clave: Cuestionario; Poblaciones vulnerables; Grupo de ascendencia Continental Africana.

\section{Introdução}

Os recursos hídricos exercem grande influência na saúde e no bem-estar humano. A má qualidade da água de consumo pode ocasionar efeitos negativos sobre a saúde de uma população, especialmente quando os indicadores socioeconômicos e ambientais já não são previamente favoráveis (Guedes et al., 2015).

A contaminação hídrica pode ocorrer por ingesta de produtos químicos usados na agricultura e na mineração e por matéria orgânica lançada a céu aberto e sem tratamento prévio. O consumo de água contaminada, seja em termos de balneabilidade ou potabilidade, e a reduzida disponibilidade de água pode promover hábitos higiênicos insatisfatórios e/ou inadequados e consequentemente o aumento do risco de adoecimento (Queiroz \& Oliveira, 2018).

As doenças relacionadas a água como amebíase, diarreia viral/parasitária e esquistossomose são responsáveis por 2,35\% das internações hospitalares totais no Brasil. Um dos principais fatores da contaminação hídrica para consumo humano está relacionado a inadequação de saneamento básico $O$ risco de adoecimento poderá ser agravado nos grupos vulneráveis e regiões mais pobres (Paiva \& Souza, 2018).

Os quilombolas que vivem em áreas rurais são mais passíveis à inadequação de saneamento básico e ao acesso insuficiente de água potável, além de possuírem menor cobertura dos serviços de saneamento básico (Queiroz \& Oliveira, 2018). Na comunidade quilombola localizada na área rural no Mato Grosso do Sul não existe infraestrutura de coleta de esgoto e a disponibilidade de água tratada (Magalhães \& Paulo, 2017). Em Juazeiro na Bahia, as análises dos padrões de potabilidade da água utilizada para consumo humano na comunidade quilombola Barrinha da Conceição foram caracterizadas como não potável, ademais, detectou-se a presença de coliformes fecais e Escherichia coli em todas as coletas (Oliveira et al., 2015).

A qualidade e a disponibilidade da água são fundamentais na redução dos casos de doenças, como a COVID-19. Este novo coronavírus (Sars-CoV-2) é uma doença considerada emergência em saúde pública pelo seu alto poder de transmissibilidade e contaminação. A higienização das mãos com água e sabão é uma das medidas mais importantes na prevenção da transmissão da COVID-19, já que a sua propagação ocorre por meio de gotículas, mãos ou superfícies contaminadas (Oliveira et al., 2020).

Além disso, a COVID-19 expõe as desigualdades existentes em uma população, seja socioeconômica, ambiental e de saúde. Deste modo, para entender as demandas de uma população é necessário compreender as especificidades de um determinado grupo populacional e as suas vulnerabilidades (Oliveira, 2021).

Informações sobre aspectos de saúde, socioeconômicos e ambientais são muito importantes na tomada de decisão direcionada e centrada nas necessidades de uma população. A coleta de dados na pesquisa em saúde envolvendo seres humanos permite a construção de conhecimentos utilizando o método científico na identificação e resolução dos problemas de saúde (Thomas et al., 2018). 
Torna-se importante, neste contexto, validar um instrumento de investigação com finalidade de analisar a associação das principais doenças relacionadas a água e a COVID-19 em comunidades quilombolas, bem como conhecer o perfil socioeconômico e ambiental deste grupo populacional.

\section{Metodologia}

A pesquisa foi aprovada pelo Comitê de Ética em Pesquisa da Universidade Tiradentes, sob o CAAE 37888620.7.0000.5371, seguindo as normativas estabelecidas pela Resolução 466/12 do Conselho Nacional de Saúde. Os especialistas que participaram deste estudo foram esclarecidos quanto ao objetivo da investigação e à natureza da coleta de dados. Aqueles que concordaram com a participação assinaram o Termo de Consentimento Livre e Esclarecido (TCLE).

Em sua versão inicial o instrumento de investigação era composto por 79 questões fechadas distribuídas em 3 (três) blocos: bloco I - sociodemográfico (15 questões), bloco II - condições ambientais e instalações sanitárias (25 questões) e bloco III- Aspectos de Saúde e Hábitos de vida (39 questões). As questões foram adaptadas a partir de um banco de questões do Instituto Brasileiro de Geografia e Estatística (IBGE), com base na Política Nacional por Amostra de Domicílios (PNAD, 2018; PNAD COVID-19, 2020), na classificação econômica da Associação Brasileira de Empresas e Pesquisa (ABEP, 2018) e no questionário de investigação epidemiológica de Calasans et al. (2018).

As questões provenientes do PNAD e ABEP estão relacionadas às informações socioeconômicas como grau de instrução, tipo de casa e a procedência da água utilizada no domicílio (ABEP, 2018; PNAD, 2018). As questões associadas a doenças relacionadas a água foram baseadas no questionário validado por Calasans et al. (2018). As questões referentes a COVID-19, tais como sintomas da síndrome gripal e da COVID-19 foram baseadas no PNAD-COVID-19 (Brasil, 2020).

O instrumento de coleta de dados construído foi submetido a validação facial de cinco especialistas nas áreas da saúde pública, ciências biológicas, ciências sociais aplicadas em saúde e ambiente. O processo foi viabilizado por correio eletrônico, através de carta convite. Os critérios utilizados na avaliação foram: comportamental, objetividade, simplicidade, clareza, pertinência, precisão, da variedade e da credibilidade. $\mathrm{O}$ uso destes critérios tem a finalidade de excluir qualquer item que esteja confuso, incompreensível, com termos vagos, duplas perguntas, jargões e/ou que remeta a um julgamento baseado em um ponto de vista pessoal (Coluci et al., 2015).

Na determinação da concordância entre os especialistas foi utilizado o Índice de Validade de Conteúdo (IVC). Este método permite analisar as questões de forma individual e posteriormente, o instrumento como um todo. Além disso, a porcentagem de concordância também foi aplicada (Alexandre \& Coluci, 2011).

Os dados foram analisados, adotando-se intervalo de confiança de $95 \%$. Considerou-se o percentual de concordância de $90 \%$ como critério de decisão sobre a pertinência do item do instrumento, ou sua modificação.

\section{Resultados e Discussão}

As questões do instrumento de coleta foram submetidas a avaliação de cinco especialistas que possuem conhecimentos e experiências em pesquisas com comunidades quilombolas. Após o processo de validação, o instrumento foi aplicado como pré-teste em uma comunidade quilombola no nordeste brasileiro com o intuito de averiguar a compreensão destes indivíduos a respeito das questões validadas pelos especialistas.

As etapas da validação do instrumento de coleta encontram-se apresentadas no infográfico da Figura 1. 
Figura 1 - Infográfico das etapas de validação de instrumento de investigação das condições de saúde, socioeconômicas e ambientais em comunidades quilombolas.

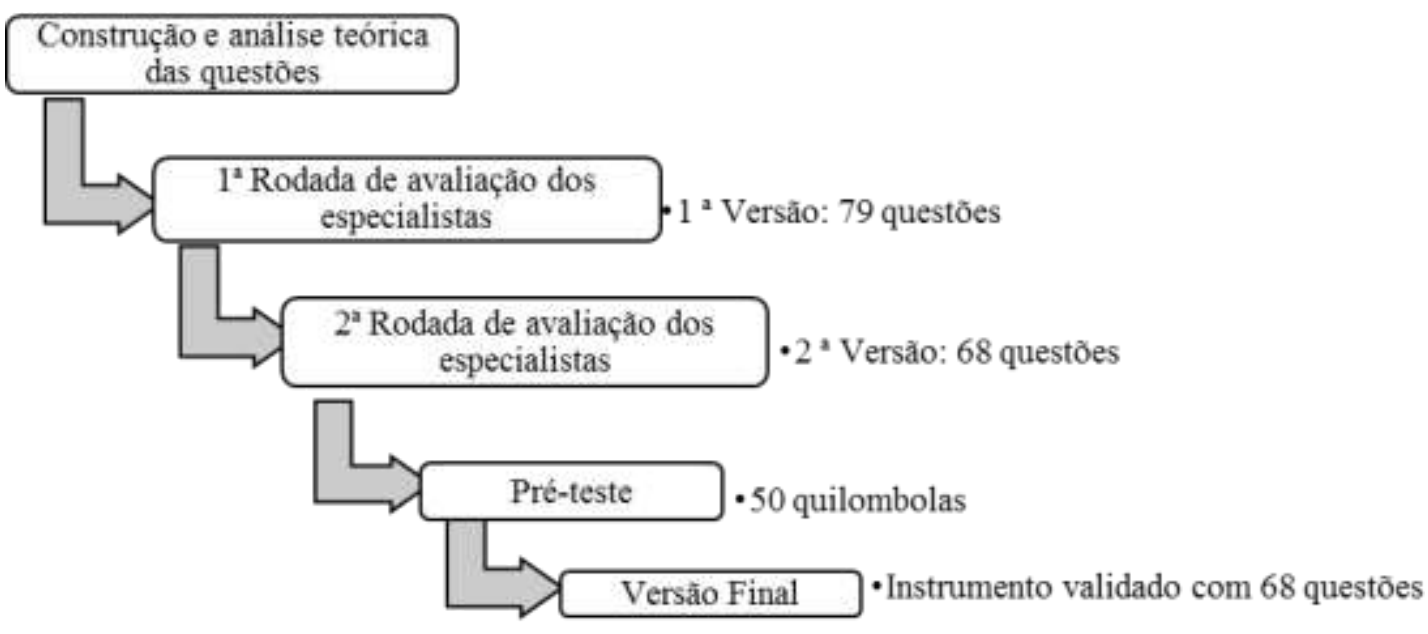

Fonte: Autores.

\section{$1^{\text {a }}$ Rodada de avaliação dos especialistas}

A primeira etapa da validação do instrumento consistiu na análise das questões baseada em critérios aplicados individualmente. Os cinco especialistas analisaram as setenta e nove questões pré-elaboradas concordando ou discordando das questões do instrumento. As análises do instrumento de investigação estão disponíveis na Tabela 1.

Tabela 1 - Análise do instrumento de investigação por especialistas na $1^{\text {a }}$ Rodada de avaliação. Sergipe, 2020.

\begin{tabular}{|c|c|c|c|c|c|c|c|c|c|c|}
\hline \multirow[b]{2}{*}{ CRITÉRIOS } & \multicolumn{2}{|c|}{ Especialista 1} & \multicolumn{2}{|c|}{ Especialista 2} & \multicolumn{2}{|c|}{ Especialista 3} & \multicolumn{2}{|c|}{ Especialista 4} & \multicolumn{2}{|c|}{ Especialista 5} \\
\hline & $\begin{array}{l}\text { Sim } \\
\%\end{array}$ & $\begin{array}{l}\text { Não } \\
\%\end{array}$ & $\begin{array}{l}\text { Sim } \\
\%\end{array}$ & $\begin{array}{l}\text { Não } \\
\%\end{array}$ & $\begin{array}{l}\mathrm{Sim} \\
\%\end{array}$ & $\begin{array}{l}\text { Não } \\
\%\end{array}$ & $\begin{array}{l}\text { Sim } \\
\%\end{array}$ & $\begin{array}{l}\text { Não } \\
\%\end{array}$ & $\begin{array}{l}\text { Sim } \\
\%\end{array}$ & $\begin{array}{l}\text { Não } \\
\%\end{array}$ \\
\hline Comportamental & 96,2 & 3,8 & 96,2 & 3,8 & 100 & - & 83,3 & 16,7 & 100 & - \\
\hline Objetividade & 96,2 & 3,8 & 96,2 & 3,8 & 100 & - & 79,2 & 20,8 & 100 & - \\
\hline Simplicidade & 95 & 5 & 95 & 5 & 100 & - & 68 & 32 & 100 & - \\
\hline Clareza & 98,7 & 1,3 & 95 & 5 & 100 & - & 76,9 & 23,1 & 98,7 & 1,3 \\
\hline Pertinência & 96,2 & 3,8 & 98,7 & 1,3 & 100 & - & 83,3 & 16,7 & 98,7 & 1,3 \\
\hline Precisão & 95 & 5 & 96,2 & 3,8 & 100 & - & 84,6 & 15,4 & 98,7 & 1,3 \\
\hline Variedade & 96,2 & 3,8 & 88,5 & 11,5 & 100 & - & 86 & 14 & 98,7 & 1,3 \\
\hline Credibilidade & 96,2 & 3,8 & 86 & 14 & 100 & - & 81 & 19 & 98,7 & 1,3 \\
\hline
\end{tabular}

Nota: Especialista 1: Mestre em Saúde e Ambiente; Especialista 2: Doutora em Saúde e Ambiente; Especialista 3: Doutora em Educação; Especialista 4: Doutor em Saúde e Ambiente e Especialista 5: Doutora em Clínica Cirúrgica. Fonte: Autores.

De acordo com os critérios estabelecidos, o instrumento de coleta obteve uma média geral de concordância de $94 \%$ observando uma heterogeneidade conforma a área profissional dos especialistas. No que diz respeito aos critérios individuais 
tem-se uma média de concordância entre os especialistas nos seguintes critérios: comportamental $(95,1 \%)$, objetividade (94,3\%), simplicidade (91,6\%), clareza (94\%), pertinência (95,4\%), precisão (95\%), variedade (94\%) e credibilidade $(92,4 \%)$. Estes critérios de concordância permitem analisar o equilíbrio e a amplitude do instrumento de coleta como um todo (Belluci \& Matsuda, 2012).

Nessa etapa, os especialistas fizeram ressalvas nos seguintes pontos: as primeiras três questões referem-se apenas à identificação e são para o controle do pesquisador. Em função disso, segundo os especialistas, não devem ser consideradas como indagações além disso, recomendaram substituição de verbos e substantivos; bem como alteração na sequência e exclusão de algumas questões.

Após esta avaliação, as três questões iniciais foram alocadas na identificação e oito questões foram excluídas, correspondendo a $10,1 \%$ do total de questões. Os especialistas relataram que essas questões poderiam demandar novas categorizações em virtude de infinitas possibilidades de respostas, presença de quadros confusos que possibilitariam a indução ao preenchimento errôneo e itens relacionados a números de aparelhos eletrônicos, domésticos e empregados que não representavam a realidade da comunidade quilombola.

Destarte, as questões foram corrigidas e adaptadas segundo as orientações dos especialistas permanecendo sessenta e oito questões no instrumento de investigação nesta etapa.

\section{2 a Rodada de avaliação dos especialistas}

Nesta etapa foram propostas sessenta e oito questões para avaliação dos especialistas. Todos os indivíduos que atuaram na primeira etapa da análise do instrumento de investigação, após confirmação quanto a nova participação, efetuaram a segunda rodada de avaliação.

De acordo com Pasquali (2010), a escala de Likert geralmente apresenta 3 ou mais pontos, em que o avaliador deve dizer se concorda, discorda ou não tem certeza sobre uma determinada afirmação. Neste estudo, utilizou-se uma escala de 4 pontos: valor 1 (discordo totalmente), valor 2 (discordo em parte), valor 3 (concordo parcialmente) e valor 4 (concordo totalmente).

A porcentagem de concordância das questões nesta etapa foi de $100 \%$ conforme a seguinte equação: \% concordância $=$ número de participantes que concordaram/número total de participantes x 100. Logo, temos: \% concordância $=5 / 5 \times 100=$ 100.

Cada questão recebeu a pontuação máxima pelos cinco especialistas, valor “4”, o que indica que as questões possuem relevância e não necessitam de alterações. Ao realizar o cálculo do índice de validade de conteúdo (IVC) para cada questão através do uso da fórmula: IVC = número de respostas 3 ou 4/ número total de respostas, obteve-se o índice de concordância no valor "1".

Segundo Souza et al (2017), o valor de concordância recomendado para a validação individual das questões deve ser no mínimo 0,8 sendo mais adequado valor acima de 0,9 . Os julgamentos dos especialistas encontram-se disponíveis na Tabela 2. 
Tabela 2 - Julgamento dos especialistas $(n=5)$ sobre as questões do instrumento de investigação. Sergipe, 2020.

\begin{tabular}{|c|c|c|c|c|c|}
\hline & \multicolumn{5}{|c|}{ JULGAMENTO } \\
\hline & \multirow[b]{2}{*}{$\mathrm{n}$} & Adequado & \multicolumn{2}{|c|}{ Adequado com alterações } & \multirow{2}{*}{ IVC } \\
\hline & & $\%$ & $\mathrm{n}$ & $\%$ & \\
\hline Questão 1 & 5 & 80 & 5 & 100 & 1,00 \\
\hline Questão 2 & 5 & 100 & 5 & 100 & 1,00 \\
\hline Questão 3 & 5 & 100 & 5 & 100 & 1,00 \\
\hline Questão 4 & 5 & 100 & 5 & 100 & 1,00 \\
\hline Questão 5 & 5 & 100 & 5 & 100 & 1,00 \\
\hline Questão 6 & 5 & 100 & 5 & 100 & 1,00 \\
\hline Questão 7 & 5 & 100 & 5 & 100 & 1,00 \\
\hline Questão 8 & 5 & 100 & 5 & 100 & 1,00 \\
\hline Questão 9 & 5 & 100 & 5 & 100 & 1,00 \\
\hline Questão 10 & 5 & 100 & 5 & 100 & 1,00 \\
\hline Questão 11 & 5 & 100 & 5 & 100 & 1,00 \\
\hline Questão 12 & 5 & 100 & 5 & 100 & 1,00 \\
\hline Questão 13 & 5 & 100 & 5 & 100 & 1,00 \\
\hline Questão 14 & 5 & 100 & 5 & 100 & 1,00 \\
\hline Questão 15 & 5 & 100 & 5 & 100 & 1,00 \\
\hline Questão 16 & 5 & 100 & 5 & 100 & 1,00 \\
\hline Questão 17 & 5 & 100 & 5 & 100 & 1,00 \\
\hline Questão 18 & 5 & 100 & 5 & 100 & 1,00 \\
\hline Questão 19 & 5 & 100 & 5 & 100 & 1,00 \\
\hline Questão 20 & 5 & 100 & 5 & 100 & 1,00 \\
\hline Questão 21 & 5 & 100 & 5 & 100 & 1,00 \\
\hline Questão 22 & 5 & 100 & 5 & 100 & 1,00 \\
\hline Questão 23 & 5 & 100 & 5 & 100 & 1,00 \\
\hline Questão 24 & 5 & 100 & 5 & 100 & 1,00 \\
\hline Questão 25 & 5 & 100 & 5 & 100 & 1,00 \\
\hline Questão 26 & 5 & 100 & 5 & 100 & 1,00 \\
\hline Questão 27 & 5 & 100 & 5 & 100 & 1,00 \\
\hline Questão 28 & 5 & 100 & 5 & 100 & 1,00 \\
\hline Questão 29 & 5 & 100 & 5 & 100 & 1,00 \\
\hline Questão 30 & 5 & 100 & 5 & 100 & 1,00 \\
\hline Questão 31 & 5 & 100 & 5 & 100 & 1,00 \\
\hline Questão 32 & 5 & 100 & 5 & 100 & 1,00 \\
\hline Questão 33 & 5 & 100 & 5 & 100 & 1,00 \\
\hline Questão 34 & 5 & 100 & 5 & 100 & 1,00 \\
\hline Questão 35 & 5 & 100 & 5 & 100 & 1,00 \\
\hline Questão 36 & 5 & 100 & 5 & 100 & 1,00 \\
\hline Questão 37 & 5 & 100 & 5 & 100 & 1,00 \\
\hline Questão 38 & 5 & 100 & 5 & 100 & 1,00 \\
\hline Questão 39 & 5 & 100 & 5 & 100 & 1,00 \\
\hline Questão 40 & 5 & 80 & 5 & 100 & 1,00 \\
\hline Questão 41 & 5 & 100 & 5 & 100 & 1,00 \\
\hline Questão 42 & 5 & 100 & 5 & 100 & 1,00 \\
\hline
\end{tabular}




\begin{tabular}{|c|c|c|c|c|c|}
\hline Questão 43 & 5 & 100 & 5 & 100 & 1,00 \\
\hline Questão 44 & 5 & 100 & 5 & 100 & 1,00 \\
\hline Questão 45 & 5 & 80 & 5 & 100 & 1,00 \\
\hline Questão 46 & 5 & 100 & 5 & 100 & 1,00 \\
\hline Questão 47 & 5 & 100 & 5 & 100 & 1,00 \\
\hline Questão 48 & 5 & 100 & 5 & 100 & 1,00 \\
\hline Questão 49 & 5 & 100 & 5 & 100 & 1,00 \\
\hline Questão 50 & 5 & 100 & 5 & 100 & 1,00 \\
\hline Questão 51 & 5 & 100 & 5 & 100 & 1,00 \\
\hline Questão 52 & 5 & 80 & 5 & 100 & 1,00 \\
\hline Questão 53 & 5 & 100 & 5 & 100 & 1,00 \\
\hline Questão 54 & 5 & 100 & 5 & 100 & 1,00 \\
\hline Questão 55 & 5 & 100 & 5 & 100 & 1,00 \\
\hline Questão 56 & 5 & 100 & 5 & 100 & 1,00 \\
\hline Questão 57 & 5 & 100 & 5 & 100 & 1,00 \\
\hline Questão 58 & 5 & 100 & 5 & 100 & 1,00 \\
\hline Questão 59 & 5 & 100 & 5 & 100 & 1,00 \\
\hline Questão 60 & 5 & 100 & 5 & 100 & 1,00 \\
\hline Questão 61 & 5 & 100 & 5 & 100 & 1,00 \\
\hline Questão 62 & 5 & 100 & 5 & 100 & 1,00 \\
\hline Questão 63 & 5 & 100 & 5 & 100 & 1,00 \\
\hline Questão 64 & 5 & 100 & 5 & 100 & 1,00 \\
\hline Questão 65 & 5 & 100 & 5 & 100 & 1,00 \\
\hline Questão 66 & 5 & 80 & 5 & 100 & 1,00 \\
\hline Questão 67 & 5 & 100 & 5 & 100 & 1,00 \\
\hline Questão 68 & 5 & 100 & 5 & 100 & 1,00 \\
\hline
\end{tabular}

Fonte: Autores.

$\mathrm{Na}$ avaliação de um instrumento com um todo por especialistas, não existe uma concordância estabelecida na literatura científica. A avaliação de um instrumento na sua totalidade pode proceder de três formas: a primeira é a média das proporções das questões consideradas relevantes pelos especialistas; a segunda é a média dos valores das questões calculadas individualmente; a terceira seria dividir o número total de questões consideradas relevantes pelos especialistas, pelo número total de questões (Alexandre \& Coluci 2011).

O instrumento de investigação possui 68 questões consideradas relevantes por especialistas. Logo, para o cálculo de concordância do instrumento integralmente, optou-se pela seguinte fórmula: questões consideradas relevantes = número total de questões/número de questões consideradas relevantes por especialistas x 100; 68/68 = 1 x 100\%.

Todas as questões avaliadas pelos especialistas nesta etapa tiveram resultados satisfatórios e neste caso, o instrumento avaliado como um todo obteve valor "1" e taxa de concordância de 100\%. De acordo com Alexandre \& Coluci (2011), quando há participação de cinco ou menos especialistas na avaliação de um instrumento, todos devem concordar para ser representativo. Contudo, segundo Coluci et al. (2015), a taxa de concordância considerada satisfatória deve indicar resultado IVC maior ou igual a 90\%. Por outro lado, a literatura também informa que na avaliação de um instrumento em sua totalidade, o IVC será considerado satisfatório quando apresentar resultado maior ou igual a 80\% (Oliveira \& Lima, 2017). 


\section{Aplicabilidade do instrumento de investigação}

O pré-teste foi desenvolvido em comunidade quilombola no leste sergipano. Incluiu-se 50 indivíduos sujeitos aleatoriamente na validação semântica do instrumento de investigação. Segundo Lakatos \& Marconi (2003), para realizar um pré-teste na validação de um instrumento, o ideal seria uma amostra de no mínimo 5\% do total de participantes, enquanto Pasquali (1998) recomenda uma amostra de 30 indivíduos da população alvo para avaliar o nível de compreensão das questões. Este estudo, optou-se pela quantidade amostral de $20 \%$ do total de participantes da pesquisa (248 indivíduos).

Nesta etapa, o instrumento de investigação contou com a presença de 3 investigadores e durante a aplicação observouse dificuldades de compreensão dos quilombolas em seis questões. Estas foram sinalizadas e adaptadas, tendo em vista a facilitação do entendimento destes indivíduos. Na Tabela 3, encontram-se disponíveis as questões adaptadas.

Tabela 3 - Questões do instrumento de investigação adaptadas, Comunidades quilombolas. Sergipe, 2020.

\begin{tabular}{ll}
\hline & Questões Adaptadas \\
\hline \multirow{2}{*}{ Gestante } & Sim ( ) \\
& Não () \\
Não se aplica ( )
\end{tabular}

Este domicílio é do tipo:

( ) Casa com vários cômodos

( ) Casa com o mesmo ambiente para diversas funções (dormir, cozinhar, fazer refeições etc.)

( ) Oca ou maloca (cabanas)

$\begin{array}{lll}\begin{array}{l}\text { Após o tratamento pode se contaminar com a } \\ \text { esquistossomose? }\end{array} & \begin{array}{l}\text { () Não } \\ \text { () Não sei }\end{array} \\ & \text { () Tosse } \\ & \text { () Falta de ar } \\ & \text { () Fadiga } \\ & \text { () Febre mesmo que referida } \\ & \text { () Dor de garganta } \\ & \text { () Congestão nasal } \\ & \text { () Dor de cabeça } \\ \text { () Dor no corpo } & \text { () Cansaço } \\ \text { Nesse último ano você/Sr.(a) já apresentou: } & \text { () Dificuldade para respirar } \\ & \text { () Diarreia } \\ & \text { () Não se aplica } \\ \text { Você já fez teste para COVID-19 } & \text { () Não } \\ & \text { () Sim, com teste rápido. } \\ & \text { () Sim, em laboratório. }\end{array}$

( ) Não recebeu resultado

Caso tenha feito o teste para a COVID-19, qual foi o

( ) Teste positivo para Covid-19

resultado?

( ) Teste negativo para Covid-19

Nota: Em itálico as frases adaptadas. Fonte: Autores. 


\section{Conclusão}

O instrumento demonstrou validade de conteúdo satisfatória e pode ser utilizado na análise das condições de saúde, com viabilidade adicional de se conhecer as variáveis do perfil socioeconômico e ambiental de comunidades quilombolas.

É importante que novos estudos possam ser desenvolvidos em comunidades vulneráveis visando a promoção da saúde e prevenção de doenças, e assim, a melhoria da qualidade de vida destes indivíduos.

\section{Referências}

ABEP. (2018). Critério de Classificação econômica Brasil. Associação brasileira de empresas de pesquisa (ABEP).

ALexandre, N. M. C. C. \& Luci, M. Z. O. (2011). Validade de conteúdo nos processos de construção e adaptação de instrumentos de medidas. Ciênc. saúde coletiva, $16(7), 3061-8$.

Bellucci Jr., J. A. \& Matsuda, L. M. (2012). Construção e validação de instrumento para avaliação do Acolhimento com Clas sificação de Risco. Revista Brasileira de Enfermagem, 65 (5), 751-7.

BRASIL. (2020). Pesquisa Nacional por Amostra de Domicílios Contínua (PNAD) COVID-19. Microdados. Instituto Brasileiro de Geografia e Estatística (IBGE).

BRASIL. (2020). Instrumentos de coleta. PNAD COVID-19. Instituto Brasileiro de Geografia e Estatística (IBGE).

Calasans, T. A. S.; Souza, G. T. R.; Melo, C. M.; Madi, R. R. \& Jeraldo, V. L. S. (2018). Socioenvironmental factors associated with Schistosoma mansoni infection and intermediate hosts in an urban area of northeastern Brazil. PLoS one, 13 (5), e0195519.

Coluci, M. Z. O.; Alexandre, N. M. C. \& Milani, D. (2015). Construção de instrumentos de medida na área da saúde. Ciênc. saúde coletiva, 20 (3), 925-936.

Guedes, G. R; Simão, A. B.; Dias, C. A. \& Braga, E. O. (2015). Risco de adoecimento por exposição às águas do Rio Doce: um estudo sobre a percepção da população de Tumiritinga, Minas Gerais, Brasil. Cadernos de Saúde Pública, 31 (6), 1257-1268.

IBGE. (2019) Características gerais dos domicílios e dos moradores 2018 (Pnad Contínua). Instituto Brasileiro de Geografia e Estatística (IBGE).

Lakatos, E. M. \& Marconi, M. A. (2003). Fundamentos de metodologia científica. 5. Ed. Editora Atlas. São Paulo.

Magalhães, F. J. C. \& Paulo, P. L. (2017). Abastecimento de água, esgotamento doméstico e aspectos de saúde em comunidades Quilombolas no Estado de Mato Grosso do Sul. Interações, 18 (2), 103-116.

Oliveira, R.; Oliveira, F. G. S.; Santos, M. A. B.; Silva, T. A. \& Moura, G, J. B. (2015). Avaliação da potabilidade da água consumida por quilombolas em Juazeiro, BA, Brasil. Opará: Etnicidades, Movimentos Sociais e Educação, 3 (4), 45-58.

Oliveira, S. K. P. \& Lima, F. E. T. (2017). Validação de conteúdo da escala de avaliação do autocuidado de pacientes com insuficiência cardíaca. Rev Rene, Fortaleza, $18(2), 148-155$.

Oliveira, A. C.; LucaS, T. C. \& Iquiapaza, R. A. (2020). O que a pandemia da covid-19 tem nos ensinado sobre adoção de medidas de precaução? Texto \& Contexto - Enfermagem, 29, e20200106.

Olvieria, R. T. (2021). A pandemia da Covid-19 e o aumento de vulnerabilidades. Reseach, Society and Development, 10 (9), 1-6.

Paiva, R. F. P. S. \& Souza, M. F. P. (2018). Associação entre condições socioeconômicas, sanitárias e de atenção básica e a morbidade hospitalar por doenças de veiculação hídrica no Brasil. Cadernos de Saúde Pública, 34 (1), e00017316.

Pasquali, L. (1998). Princípios de elaboração de escalas psicológicas. Revista Psiquiatria Clínica, 25 (5), $206-213$.

Pasquali, L. (2010). Instrumentação Psicológica: Fundamentos e práticas, 165-198.

Queiroz, T. M. \& Oliveira, L. C. P. (2018). Qualidade da água em comunidades quilombolas do Vão Grande, município de Barra do Bugres (MT). Engenharia Sanitária e Ambiental, 23 (1), 173-180.

Souza, A. C.; Alexandre, N. M. C. \& Guirardello, E. B. (2017). Propriedades psicométricas na avaliação de instrumentos: avaliação da confiabilidade e da validade. Epidemiologia e Serviços de Saúde, 26 (3), 649-659.

Thomas, D. B.; Oenning, N. S. X. \& Goulart B. N. G. (2018). Aspectos essenciais na construção de instrumentos de coleta de dados em pesquisas primárias de saúde. Rev. CEFAC, 20 (5), 657-664. 